\title{
Funciones del Secretario particular de los Presidentes de las Corporaciones locales
}

\author{
por \\ JULIAN CARRASCO BELINCHON \\ Secretario de r." categoría de Administración Local
}

SUMARIO: I. Introduccion.-II. Funciones del Secretario particular: 1. Planteamiento. 2. Correspondencia. 2-1. Planteamiento. 2-2. Recepción. 2-3. Despacho. 2-4. Remisión. 2-5. Clasificación y archivo de la correspondencia. 3. Visitas. 3-1. Planteamiento. 3-2. Recepción. 3-3. Atención durante ia espera. 3-4. Entrevista. 3-5. Despedida. 4. Teléfono. 4-1. Planteamiento. 4-2. Cómo debe actuar el Secretario con el teléfono. 4-3. Qué tiene que hacer el Secretario para que el teléfono sea un instrumento útil para el Presidente. 5. Dirección de la Secretaría como unidad administrativa.

6. Programación del Presidente.-III. Conclusiones.

\section{INTRODUCCION}

El número 15 del artículo 121 del vigente Reglamento de Organización, funcionamiento y régimen jurídico de las Corporaciones locales, reconoce como atribución de los Alcaldes el «nombrar y remover en los Municipios de más de $\mathbf{1 5 . 0 0 0}$ habitantes y en las capitales de Provincia, si lo estima necesario, un Secretario particular, dotándolo con cargo al presupuesto».

Si bien en la enumeración de las atribuciones del Presidente de la Diputación provincial, contenida en el artículo 168 de dicho Cuerpo legal, no existe precepto similar, no obstante éste tiene también la facultad de nombrar Secretario particular, por la analogía existente entre los Presidentes de las Corporaciones locales.

Ahora bien, como las funciones del Secretario particular no están reglamentadas legalmente, surgen a veces dudas sobre cuá- 
les son, e incluso pueden suscitarse interferencias con las que corresponden al titular de la Secretaría general, y ello no por razón de la competencia de las personas que ostentan uno y otro cargo, sino precisamente por razón de la confianza que en ellas deposite el Presidente de la respectiva Corporación.

En principio, y desde el punto de vista personal, es evidente que siendo nombrado y removido libremente el Secretario particular por el Presidente, éste tendrá más confianza en un Secretario particular que en el Secretario general que, normalmente, es anterior al propio Presidente, y no puede ser libremente removido por éste.

Ante esta situación, y teniendo en cuenta que las funciones del Secretario general están definidas legalmente en los artículos 140 al 145 del vigente Reglamento de Funcionarios de Administración local (1), con lo cual está delimitado su campo privativo de competencia, que en ningún caso deberá ser invadido por el Secretario particular, es conveniente examinar cuál puede ser el cometido de éste.

Precisamente el objetivo del presente trabajo es el de exponer, desde el punto de vista de la técnica de la organización, cuáles son las funciones que corresponden al Secretario particular, las que no invaden, sino que complementan, a las que pertenecen al titular de la Secretaría general.

\section{FUNCIONES DEL SECRETARIO PARTICULAR}

\section{Planteamiento.}

Como en otra ocasión pusimos de relieve (2), el Secretario particular tiene normalmente que desempeñar las siguientes funciones esenciales:

(1) En nuestro trabajo «La Jefatura administrativa en las Corporaciones locales», publicado en el número 110 de esta misma RevisTA, analizamos detenidamente dichas funciones, y al mismo nos remitimos.

(2) «El Secretario particular, como primer auxiliar del jefe», publicado en $\ll D$. A.» número 36 , diciembre 1960. Artículo que complementa ideas de las que aqui esbozamos, y al que nos remitimos para evitar repeticiones. 
- Recepción, preparación, despacho, envío, clasificación y archivo de la correspondencia no oficial.

- Recepción, atención, introducción y despedida de las visitas.

- Atención al teléfono.

- Dirección de la Secretaría particular como unidad administrativa.

- Programación del trabajo respectivo del Presidente (3).

En definitiva, el Secretario particular es el primer colaborador del jefe en las tareas «no directivas». Es decir, que el Secretario particular de los Presidentes de las Corporaciones es su principal auxiliar, pero en las tareas «no directivas», pues en éstas su colaborador fundamental es el Secretario general.

Están, pues, en la realidad, delimitados claramente los campos, y si se respetan mutuamente, obtienen grandes beneficios ambos.

Ahora bien, este respeto no significa igualdad, pues el Secretario general, en cuanto jefe de personal, es el superior del Secretario particular, aunque no su jefe inmediato.

\section{CORRESPONDENCIA.}

2. 1. Planteamiento.

Comprendemos bajo tal denominación el conjunto de comunicaciones escritas, no oficiales, que recibe, despacha y remite una Secretaría particular.

En base a esta idea de la correspondencia, podemos fijarnos en cuatro momentos: recepción, despacho, remisión y clasificación y archivo.

(3) Esta definición de funciones del Secretario particular ha tenido, con posterioridad, confirmación oficial en el Reglamento provisional de Gobiernos civiles de 24 de julio de 1961, en cuyo artículo 29 se dispone: $\$$ El Gobernador civil podrá designar libremente un Secretario particular como auxiliar cualificado para las actividades de esta índole, cuyas misiones principales serán las siguientes:

1. Recepción, preparación, despacho, envio, clasificación y archivo de la correspondencia no oficial.

2.: Régimen de audiencias y visitas personales al Gobernador.

3. La dirección de la Secretaría particular, como unidad administrativa.» 


\section{2. Recepción.}

Las principales normas prácticas que se propugnan, las podemos sintetizar en las siguientes:

Apertura del correo:

- Abrir los sobres con cuidado para no romper su contenido.

- Extraer este contenido, cuidando de que no quede nada dentro y comprobando que viene todo lo que se dice que se envía.

- Estampar la fecha en que se recibe para evitar posibles dudas posteriores sobre el día en que llegó.

Clasificación:

El criterio que debe presidirla es el de evitar al Presidente trabajo innecesario. En consecuencia, se debe dividir el correo en dos grandes grupos:

- El que puede pasar directamente al Presidente.

El Secretario debe prepararle, uniendo a los documentos recibidos, los antecedentes que se posean sobre el asunto; destacar en la comunicación recibida las frases o párrafos más importantes; adjuntando notas aclaratorias sobre la cuestión que se suscite y colocando la correspondencia por orden de importancia, procurando anteponer las cartas favorables a las desfavorables.

- El que no debe pasarse directamente al Presidente.

Este grupo comprende el correo que puede contestar directamente el Secretario y el que debe ir a otra persona, por ser la que realmente puede contestar al asunto que se plantea.

\section{3. Despacho.}

Podemos distinguir: el correo que puede contestar directamente el Secretario, ya firmándolo el Presidente, ya firmándolo él, y el correo que debe contestar el Presidente. 
Con respecto al primero, se recomienda rapidez en su despacho, exactitud en su contenido y cordialidad en la expresión (4).

En cuanto al segundo, se pueden diferenciar dos momentos importantes: dictado y mecanografiado.

Respecto al dictado de la correspondencia debe procurarse:

Tener señaladas horas fijas para dictar; tener establecida una llamada especial cuando se desea que vaya el taquígrafo preparado; dictar con claridad y firmeza; indicar los signos de puntuación importantes y entregar al taquígrafo las cartas a medida que las vaya contestando (5).

Por su parte, el taquigrafo debe: comenzar el dictado de cada día en una página nueva, reseñando la fecha al comienzo; sentarse cómodamente frente al que dicta; no distraer la atención de éste; pedir aclaraciones en caso de duda, inmediatamente o al final, se-

(4) En toda comunicación escrita-advierte Cecilia ToRmo DE ARIASse pueden distinguir dos aspectos: el externo, que consiste en la estética de sus proporciones, y el interno, o sea, la expresión de su contenido.

El cuidado en la confección mecanográfica de un escrito asegura a éste el 50 por 100 de su éxito. La simetría y la nitidez de la estructura tiene tanta importancia como la redacción breve y clara.

Para que un escrito tenga poder persuasivo, necesita de ambos iactores; es difícil considerarlos separadamente; si uno de ellos se descuida, tiende a ignorarse el otro; si la expresión del contenido no es suficientemente clara, concisa, razonable, cortés y agradable, entonces la primera impresión causada por el aspecto externo, por la estética y simetría de las properciones, será solamente una máscara superficial que tiende a desaparecer. (Temas de Secretaría principal, Esapac, San José de Costa Rica, 1958, pág. 43.)

(5) Todo el que aspire a saber dictar con eficacia-afirman C. L. LitrueField y R. L. PETERson-deberá prestar una atención rigurosa a los siguientes principios básicos, y se esforzará concienzudamente en aplicarlos:

1. Determinar la razón y el objeto de la carta que se va a redactar: considerar que el objetivo tiene naturaleza doble, es decir, que tiene consecuencias inmediatas y remotas.

2. Planear la carta: conocer las ideas que se desean exponer y disponerlas siguiendo un orden lógico de sucesión.

3.- Usar el lenguaje natural de la conversación.

4.- Suprimir toda posibilidad de confusión.

5. Ser breve, pero sin sacrificar la suficiencia y la cortesia por causa de la brevedad.

6. Ser siempre cortés, teniendo en cuenta los sentimientos del lector. (Organización de oficinas, página 237, Méjico, 1960.) 
gúr prefiera la persona que dicta; tomar el nombre exacto y cirección completa del destinatario, y llamar la atención de los defectos de lo dictado, para que pueda corregirse.

En el momento de pasar a máquina lo dictado, es conveniente: tener a mano la carta que se contesta, para poder aclarar cualquier punto conf̂so en lo tomado; emplear la forma de carta por la que el Presidente tenga preferencia; lograr una presenıación agradable; emplear una carta para cada asunto y revisar las cartas, según se van escribiendo, para tenerlas dispuestas por si el Presidente las pide (6).

Al transcribir el texto, se ha de tener presente, como afirma VICÉNS CARRIó, las siguientes directrices: concepto estético de la distribución mecanográfica; fidelidad de redacción; corrección ortográfica e idiomática, y pulcra presentación (7).

Las cartas, nos dicen NiELANDER y MrLLER, deben ser repasadas con todo detalle. Toda carta debe ser vuelta a leer antes de ser firmada, pues incluso la mejor de las mecanógrafas puede tener un momento de descuido. La colocación equivocada de los puntos

(6) Aspecto que parece secundario, pero que, sin embargo, tiene su importancia, es el de la «posición correcta» para escribir a máquina, para disminuir el cansancio de la persona que escribe. «Posición correcta» que, según Cecilia ToRmo DE ARIAS, requiere:

- Mantener la cabeza erecta, pero relajada, vuelta ligeramente a la derecha; fija la vista en el texto.

- Mantener los dedos curvados y cerca de las teclas, con las manos en el mismo declive del teclado.

- Mantener los brazos y codos en una posición descansada, dejando que los antebrazos se inclinen naturalmente, con las manos en el teclado.

- Colocar el texto a la derecha de la máquina de modo que al devolver el carro no se estorbe la línea de lectura.

- Mantener ambos pies de lleno sobre el piso, ligeramente apartados. Uno de ellos puede estar ligeramente delante del otro.

- Ajustar la altura de la silla con respecto a la altura de la máquina de escribir, para permitir una escritura más cómoda.

- Procurar que la silla esté directamente frente a la máquina.

- La altura de la silla debe medir la distancia desde la parte interior de la rodilla al suelo, permitiendo que la pierna izquierda se doble en un ángulo recto, sin esfuerzo alguno de los músculos de las partes superior e inferior de la pierna. (Ob. cit., pág. 80.)

(7) Formación del oficinista y de la secretaria, págs. 181-2, Barcelona, 1948. 
y comas, las mayúsculas fuera de lugar, las abreviaturas erróneas y los márgenes incompletos pueden dar al traste con lo que de otra forma sería una carta efectiva (8).

\section{4. Remisión.}

En este momento se debe, en primer lugar, comprobar si todas las cartas están firmadas y van acompañadas de los anexos que en las mismas se dice que se envían; en segundo término, realizar las correcciones que procedan, tanto en el original como en la copia y, finalmente, examinar si coinciden las señas del sobre con la dirección de la carta.

2. 5. Clasificación y archivo de la correspondencia.

Preocupación constante del Secretario debe ser el tener siempre clasificada y archivada la correspondencia despachada.

Para ello debe tener en cuenta, como nos dice A. Brauman, cuatro reglas sencillas y de gran utilidad (9):

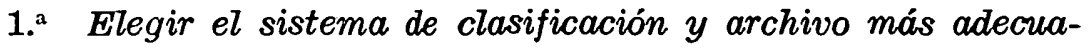
do a nuestras necesidades.

Elección que impone el previo conocimiento de las ventajas e inconvenientes de cada uno de los sistemas usuales de clasificación. Sistemas que, como es sabido, son o alfabéticos-nominativo, ideológico y geográfico-, o numéricos—numérico simple, terminal, decimal y cronológico-, o mixtos.

En principio, cualquiera de estos sistemas es útil, pero se ha de considerar detenidamente cuál o cuáles son más convenientes en cada caso concreto, por servir mejor a nuestras necesidades (10).

(8) Relaciones públicas, pág. 285. Barcelona, 1958.

(9) Guta de la archivera, pág. 50. Barcelona, 1959.

(10) Cada sistema de clasificacion-pone de relieve Italo BALDINI-es especialmente recomendable para aplicaciones determinadas y no es pesible decir, en términos absolutos y desde un punto de vista general, cuál sea la clasificación mejor y más aconsejable.

En consecuencia, el método de clasificación de los papeles de archivo tiene que estudiarse detenidamente en cada caso específico y nunca hay que 
En términos generales, normalmente, no se aplica un únicu sistema, sino que en la práctica se suelen combinar. Así es frecuente reunir los documentos por asuntos-ideológico-, colocarlos dentro de cada carpeta por fecha-cronológico-y ordenar alfabética o numéricamente las carpetas-nominativo o numérico-.

Es decir, que en la realidad se observa un sistema mixto: ideológico-cronológico-nominativo o numérico.

Lo que significa, en conclusión, que la elección se reduce, fundamentalmente, a inclinarse por un sistema nominativo o por uno numérico. De ambos, parece más útil en las Secretarías particulares el numérico, por cuanto ofrecen más posibilidades de desarrollo ulterior, destaca cualquier error o ausencia y permite más reserva con respecto a su contenido, pues sólo la persona que lo tiene a su cargo puede saber en cada caso dónde están archivados los documentos.

Estas ventajas compensan el inconveniente de requerir siempre la existencia y manejo de un repertorio alfabético que permite conocer el número asignado a cada documento o asunto.

En cuanto a los sistemas de archivo: horizontal, vertical-de canto, normal, con suspensión, con visibilidad lateral por suspensión y con visibilidad lateral sin suspensión-, rotatorios y colgantes; ofrecen cada uno sus ventajas y presentan sus inconvenientes y además de precisar cada uno el mobiliario adecuado, imponen que se consideren previamente las posibilidades que brindan, según cuál sea el material que se vaya a archivar.

El sistema horizontal únicamente se recomienda para la conservación ordenada de material de oficina: cartas, sobres, etc.

limitarse a acudir a uno de ellos porque haya sido aplicado en circunstancias análogas o por otras razones que no sean las derivadas de un detallado estudio de las exigencias de la empresa.

Al efectuar la elección, se tendrá en cuenta:

1. Que el sistema adoptado debe ser el más simple posible y tiene que dejar el menor número de casos dudosos.

2. Que debe simplificar al máximo la colocación de los documentos.

3. Que hay que considerar las necesidades futuras, para no verse obligados. a modificar posteriormente el ordenamiento. (El Archivo, págs 22-3. Barcelona, 1960.) 
El vertical de canto es el adecuado para la colocación de libros y carpetas de cierta consistencia.

Los sistemas vertical normal y con suspensión, son apropiados para el archivo de carpetillas abiertas y fundas.

En tanto que los rotatorios, por la gran viabilidad que ofrecen, son los más indicados para la conservación de fichas.

Finalmente, los colgantes son muy apropiados para la conservación de correspondencia no voluminosa.

\section{2." Archivar al día.}

Ahora bien, esta norma quizá sea más práctica si en vez de archivar al día, se clasifica cotidianamente y se archiva al final de cada semana (11).

Con ello se obtienen dos ventajas: tener en todo momento ordenada la documentación, con lo cual se encuentra inmediatamente el escrito que se busca; y hacer que en lugar de extraer y colocar varias veces las mismas carpetas durante la semana, sólo sea preciso extraerlas y colocarlas una vez.

Claro que para lograr estas ventajas es necesario disponer de varias carpetillas que nos permitan ir sobre la marcha clasificando el material pendiente de archivo.

\section{3. $\quad$ Dividir siempre del mismo modo la documentación a clasificar.}

Es decir, que en las operaciones de selección se efectúen siempre las mismas divisiones. Por ejemplo, si el sistema de clasificación que se sigue es el nominativo, la división puede ser: A-C, D-G, H-M, N-R y S-Z.

Si siempre fraccionamos la correspondencia en cinco montones, y cada uno de éstos comprende de manera constante la documenta-

(11) Cualquiera que sea la modalidad que se adopte, hay que archivar cuidadosanente. Un papel erróneamente archivado-manifiestan Charles B. HICKS e I. PLACE-puede considerarse perdido y no puede encontrarse fácilmente. En un archivo de correspondencia, por ejemplo, colocar el material detrás de la guía adecuada dentro de la carpeta correspondiente, con la îcha más reciente delante. Dejar visible el encabezamiento o título. (Orgailí:ación de oficinas, pág. 608. Barcelona, 1960.) 
SECRETaRio Particllar de LOS PRESIDENTES DE LAS CORPORACIONES 185

ción cuyas iniciales sean las indicadas, lograremos realizar el trabajo con gran rapidez y exactitud.

\section{4. ${ }^{\mathrm{a}} \quad$ Hacer fácil y cómoda la brisqueda.}

Para lo cual, se recomienda: referenciar los documentos; poner un nombre, un título o un número a cada carpeta, y ello en sitio bien visible; emplear carpetas de distintos colores, y dejar siempre constancia de todo documento extraído del archivo.

\section{Visitas.}

\section{1. Planteamiento.}

Una de las misiones fundamentales del Secretario es la de atender las visitas, procurando que éstas no desborden al Presidente, de tal forma que no quede convertido en «un amable conversador que invierte su tiempo en escuchar problemas y peticiones, $y$ en repartir saludos y sonrisas».

El Secretario, respecto a las visitas, no puede ser un valladar infranqueable, ante el que fracasan todos los intentos de aquéllas de llegar al Presidente. El Secretario tiene que canalizar, no impedir todas las visitas. Pues, en otro caso, provocaría el aislamiento total del Presidente y, con ello, determinaría la pérdida de muchas posibilidades de acción del mismo.

En definitiva, en este aspecto, el Secretario debe ser el vigilante del tráfico que conduce al Presidente, no el disco rojo que impide éste; ha de saber cuándo ha de dejar el paso libre, cuándo lo tiene que retener, cuando desviarlo a otro destino $y$, en fin, también cuándo debe impedirlo drásticamente.

Por otra parte, el Secretario tiene que completar la actitud del Presidente con respecto a las visitas y audiencias. Si el Presidente tiene predisposición a recibir a todos los visitantes, el Secretario tiene que «filtrarlos» para evitar que ocupen a éste todo su tiempo. En cambio, si el Presidente tiene la tendencia contraria, el Secretario ha de procurar que reciba a las que estime de interés.

Las normas prácticas que se sugieren con respecto a la orga- 
nización del régimen de visitas, se pueden concretar en los siguientes momentos: recepción, atención, entrevistas y despedida.

Propugnando, como directriz básica, el sugerir a los visitantes la conveniencia de solicitar con anticipación hora para celebrar la entrevista que se desea, con lo cual se les puede atender mejor, pierden menos tiempo en la espera y el Presidente puede organizar su trabajo y dedicarles el tiempo que precisen, sin prisas.

\section{2. Recepción.}

El Secretario debe causar una grata impresión al que llega y, para ello, debe: saludar afectuosamente al visitante, invitarle a que se siente, rogarle que exponga su pretensión, averiguar su nombre y la relación que tiene con el Presidente, ofrecerse a resolverle su problema, si es posible, e indicar las posibilidades que el Presidente tiene de recibirle y tiempo probable de espera y, en caso negativo, sugerirle otro momento, o hacerle ver la conveniencia de que solicite telefónicamente una entrevista para fecha posterior (12).

3. 3. Atención durante la espera.

El Secretario debe procurar: que el visitante se encuentre cómodo; se halle entretenido, facilitándole revistas, periódicos, etcétera, conversar con él, si éste lo desea, y puede ser útil el diálogo para averiguar la personalidad del mismo y los objetivos que persigue, y no discutir con el visitante, ni perder el dominio de sí mismo por cualquier manifestación inoportuna que éste pueda hacer.

(12) En este sentido merece destacarse que, según STICKNEY, FLood, Horton y WeIL, hay que cuidar en la recepción:

1. Levantarse y adelantarse a recibir al que llegue. No hay cosa más humillante para un visitante que el ser ignorado, aunque sea por un momento.

2. Recibirlo con una sonrisa. Si el asunto que lo lleva es importante, él quedará bien impresionado y, si no, le será al Secretario más fácil librarse de él.

3. Procurar obtener la mayor información posible acerca del visitante y del motivo de su visita, a fin de poder clasificarlo y tratarlo de acuerdo. (Preparación para Secretarias y oficinistas, págs. 97-98. Méjico, 1960.) 


\section{4. Entrevista.}

Durante la entrevista, el Secretario, en principio, no debe interrumpirla. No obstanıe, aquélla pueda prolongarse demasiadc, y entonces el Secretario debe, o entrar en el despacho o utilizar el teléfono-según preîiera el Presidente-para «recordar»a éste, que «el señor Gómez le está esperando», o que «a las doce tiene tal reunión», etc. Con lo cual la visita comprende, o debe comprender, que tiene que marcharse, o que le quedan aún unos breves instantes, en el caso de que el Presidente contestase: «diga que dentro de diez minutos estoy con él o estoy allí».

$Y$ si la visita no entiende, o no quiere entender, la indicación y continúa, el Secretario, con corrección, pero con resolución, debe insistir en los recordatorios.

\section{5. Despedida.}

El Secretario debe tener siempre presente, que si el visitante fué recibido correctamente y estuvo cómodo mientras esperaba, se marchará agradecido, independientemente del resultado de su gestión.

Por otra parte, no debe olvidar que tan importante como saber recibir es saber despedir, pues aunque la despedida sea rápida, como es el último contacto del que se va, el modo de tratarle deja huella en su ánimo.

\section{Teléfono.}

\section{1. Planteamiento.}

El uso de las comunicaciones telefónicas, por su rapidez, sencillez, economía y utilidad, se va ampliando cada vez más, sustituyendo en lo posible a las comunicaciones escritas, que son más lentas, más complejas y, normalmente, más caras.

El teléfono se ha revelado como un instrumento excepcional de relación, que hace desaparecer las distancias y que permite la comunicación personal entre individuos que se hallan en lugares diferentes.

Ahora bien, si el teléfono entraña infinitas posibilidades, no 
siempre aprovechadas, sin embargo presenta también una serie de exigencias, las que si no se tienen en cuenta y se observan meticulosamente, hacen que aquéllas se reduzcan considerablemente y que, incluso en algún caso, provoquen problemas perjudiciales.

\section{2. Cómo debe actuar el Secretario con el teléfono.}

Es un hecho cierto que muchas personas se forman un juicio favorable o desfavorable de la Corporación de que se trate, según como fueron atendidas telefónicamente.

En consecuencia, es esencial prestar atención a la relación telefónica. Cuidado que en especial ha de tener el Secretario, pues si una desatención por parte de un subordinado resulta molesta, alcanza el grado de imperdonable si el causante es el propio Secretario.

De aquí el que éste requiera, no sólo conocer las normas del uso correcto, sino que, sobre todo, las aplique constantemente.

La atención, la preocupación sincera por los problemas del interlocutor, el auténtico deseo de servir, la simpatía, deben ser las notas relevantes de la conducta del Secretario (13).

Por otra parte, resulta muy útil, aunque no siempre se posea, el tener memoria auditiva, que permite identificar a las personas antes que ellas se presenten, y poderlas saludar por su nombre.

Finalmente, se aconseja que se tenga la costumbre de anotar

(13) A este respecto, la Organización Paul Planus recomienda saber escuchar; escuche atentamente-dice-al interlocutor mientras le expone el asunto de que se trata, le solicita cualquier cosa o le formula una pregunta. Piense sobre lo que le está diciendo, para evitar enojosas repeticiones de conceptos; además, el interlocutor puede creer que no ha prestado usted atención a sus palabras o, lo que es peor, que encuentra usted torpe su manera de expresarse. Esto produce una pésima impresión.

Sin embargo, no deje nunca que una informacion o un dato importante queden para usted en una nebulosa ni aun con el pretexto de que no quiere usted molestar o causar una mala impresion haciendo repetir conceptos al interlocutor. No le dé nunca a entender que, por su culpa, usted no ha comprendido bien. Busque un pretexto que salve el prestigio del comunicante o invoque, por ejemplo, un ruido exterior e inoportuno. (El arite de hablar por teléfono, pág. 41. Barcelona, 1958.) 
inmediatamente los extremos que lo requieran de la conversación mantenida, en especial:

- Cuando tengan que transmitirse al Presidente.

- Cuando deban trasladarse a otra persona.

- Cuando se refieran a actividades que deban llevarse a cabo ulteriormente.

4. 3. Qué tiene que hacer el Secretario para que el teléfono sea un instrumento útil para el Presidente.

En este sentido, se recomiendan las siguientes directrices:

1. ${ }^{a} \quad$ Filtrar hábilmente las llamadas dirigidas al Presidente para conseguir que lleguen a éste únicamente las importantes.

2. ${ }^{a} \quad$ Suplir, siempre que sea posible, al Presidente atendiendo directamente todos aquellos casos en que pueda resolver por sí el Secretario.

3. ${ }^{a}$ Desviar, en los supuestos que proceda, la llamada al Presidente, a la persona que mejor pueda atender al interlocutor.

4. ${ }^{2}$ Acostumbrar a las personas que llaman, a que lo hagan en horas determinadas en las que podrá el Presidente atenderles mejor.

Quizá resulte aún más útil invitarlas a que faciliten el número de su teléfono, para ser llamadas en el momento en que mejor pueda ocuparse de ellas el Presidente.

Esta última variante es de gran utilidad..., siempre y cuando realmente se llame después, pues en otro caso, se convierte en desatención al comprobar que fué una argucia para cortar la comunicación.

5. ${ }^{a} \quad$ Averiguar, en los casos que se pueda, el motivo de las llamadas, para anticipárselo al Presidente y que éste esté preparado el iniciar el diálogo. En este sentido, se ha puesto de relieve que el que llama puede colgar en cualquier momento, terminando así la conversación, por lo que se debe ser particularmente persuasivo a fin de detener la información deseada sin ocasionar que una persona importante cuelgue el teléfono con disgusto (14).

(14) Stickney, Flood, Horton y Weil, ob. cit., pág. 109. 
6. Cuando el Presidente está ausente, y no se puede atender directamente la llamada, el Secretario debe enterarse: de la personalidad del que llamó, del motivo de la llamada y de dónde y cuándo se le puede llamar.

Tomando nota de todo ello, y comunicándolo a aquél en cuanto llegue.

5. Dirección de la Secretaría como unidad administrativa.

El Secretario es el jefe de la Secretaría particular y, como todo jefe, tiene que organizar y dirigir ésta y disciplinar, impulsar y formar al personal que la integra.

En el primer aspecto, teniendo en cuenta las tareas que debe realizar ésta, tiene que estructurar los distintos puestos de trabajo, en base a los principios de división de funciones y de uniformidad, continuidad y concentración de actividades.

Como normalmente el número de funciones a realizar será superior al de subordinados con que cuente, precisará determinar estas funciones esenciales $y$, después, distribuirlas entre éstos de conformidad con los tres principios últimamente enunciados.

Los puntos principales que en la organización ha de tener en cuenta el Secretario, son los siguientes:

1. Reservarse en todo caso la clasificación y preparación de la correspondencia; la recepción, atención y despedida de las visitas, y el atender ciertas llamadas telefónicas.

2. Establecer la rotación entre los distintos puestos de trabajo, para adiestrar a todos los subordinados en las diferentes tareas.

3. Institucionalizar las distintas actividades, de forma que puedan realizarse todas y cada una de ellas, cuando él esté ausente.

4.? Tener designado y adiestrado un sustituto que le reemplace en los casos que proceda.

5. Contar en todo momento con los medios materiales requeridos para llevar a cabo su labor.

En el segundo aspecto, el Secretario tiene que programar el trabajo de sus subordinados y el propio; llevarlo a la práctica; 
SECRETaRIo PARTICULAR LE LOS PRESDENTES DE LAS CORPORACYONES 191

armonizar las diversas actividades y supervisar su desarrollo (15).

La planificación, según R. W. FaIRBanks, consiste en aislar los resultados finales esenciales para la oficina, en conocer las áreas que más gastos ocasionen en la obtención de tales resultados y en crear el sistema adecuado para conseguir aquellcs resultados de la forma más directa posible y con el mínimo esfuerzo humano (16).

Una faceta interesante, si bien a primera vista secundaria de este aspecto, es la de cuidar el orden y pulcritud de la Secretaría: en primer lugar, para fomentar el ambiente humano de los que en ella trabajan $\mathrm{y}$, en segundo término, para proporcionar una impresión grata al visitante.

En este sentido, el Secretario no puede perder de vista los factores de índole material como determinantes de un ambiente humano (17). A este respecto, la experiencia ha acreditado lo fecundo que resulta que en la variación de dichos factores participen activamente los afectados, sugiriendo los cambios que en su opinión deben introducirse.

En el tercer supuesto, el Secretario ha de juzgar más con los estímulos que con las reprimendas, estando siempre dispuesto a escuchar cualquier reclamación, investigarla y a darle la solución oportuna.

Si cabe sacar el máximo partido posible de sus subordinados, sin acosarlos, si sabe corregir errores, evitar con energía negligencias y faltas de tacto, en fin, gobernar con firmeza y suavi-

(15) El jefe de Secretaría aumentará la eficacia de su trabajo-se recomienda en el «Handbook Secretarial Practice» deI I. I. C. A.-mediante el planeamiento de su labor; ahorrará así tiempo y multiplicará el rendimiento.

Despachará a diario con su jefe, en las primeras horas de la jornada; le suministrará un orden del día (asuntos urgentes, visitas, etc....), y se informará entonces de cuanto interese al trabajo del dia, obteniendo instrucciones sobre asuntos que serán delegados a otros funcionarios.

Prevea, pues, las tareas y planee metodicamente su ejecución; calibre la finalidad, valor, alcance $\mathbf{y}$ objetivos de cada tarea, y clasifíquelos según su importancia real; sistematice, en fin, el trabajo de rutina. (Tomado de «D. A.», núm. 4, abril 1958.)

(16) La automación de oficinas, pág. 111. Barcelona, 1960.

(17) Vid. nuestro artículo citado «El Secretario particular como primer auxiliar del jefe». 
daa a la vez, su influencia personal logrará-destaca F. L. MAYENBERG-más y mejores resultados que todas las facilidades técnicas con que pueda estar equipada la Secretaría (18).

En el cuarto aspecto, el Secretario ha de gozar de prestigio ante sus subordinados; los ha de inspirar confianza, $y$ ha de lograr su afecto, si desea convertirlos en un equipo integrado y eficiente.

El Secretario tiene que ser el primero en el trabajo y en la responsabilidad, si quiere, realmente, ser el primero en el grupo.

$\mathrm{Y}$, por último, se ha de preocupar, en lo posible, de perfeccionar a los que están bajo su mando, tanto en el ámbito profesional, como en el humano y social.

\section{Programación del trabajo del Presidente.}

El Secretario no sólo tiene que planear su propio trabajo y el de sus subordinados, sino que también ha de preocuparse de encauzar el de su Presidente, para impedir que se vea envuelto en el cúmulo de actividades a realizar.

Puede resultar extraño el pensar que un inferior intervenga en la ordenación y preparación del trabajo de su superior. Y, sin embargo, la realidad impone esta pequeña paradoja.

Sólo apoyándose en el Secretario, y contando con su ayuda, puede salir adelante con éxito el Presidente, desentendiéndose de los detalles, encargando la ejecución material y utilizando el material preparado por el Secretario.

¿Cómo se puede efectuar esta programación? Durante el despacho diario, en el que se ordena la actividad de ambos, tanto para la jornada presente como para la actuación futura, trazando sus líneas generales.

El papel del Secretario, en la planificación del trabajo del Presidente, adquiere un acusado relieve:

- Recordando a éste las actividades que debe realizar en cada período de tiempo.

- Preparando y facilitando las mismas.

(18) Dirección y administración industrial, pág. 48. Barcelona, 1959. 
SECRETARIO PARTIClLAR DE LOS PREsidentes DE LAS CORPORACIONES 193

- Procurando que a cada una de ellas pueda dedicar el tiempo que su importancia demanda.

- Impidiendo que simultáneamente tenga que atender a más de una tarea.

- Eliminando la posibilidad de que se le acumulen y retrasen los asuntos cuya resolución le incumbe, por falta de tiempo.

Ahora bien, el Secretario únicamente podrá llevar a cabo con eficacia esta función esencial de programación si está muy compenetrado con su superior y, además, si en todo momento conoce las actividades que éste ha de realizar y si oportunamente las recuerda.

La primera exigencia es de índole personal, por cuanto la compenetración se basa en la armonía-no siempre identidad-de mentalidades y en la confianza mutua.

La segunda exigencia descansa tanto en la constante comunicación que el Presidente dé de las actividades a realizar, como en la capacidad del Secretario para detectarlas y preverlas.

Mientras que la última se funda en la adecuada organización de los recordatorios: memorándums, agendas, etc.

Sólo en base a esta triple necesidad, el Secretario puede ser a la vez el primer colaborador del Presidente y su principal planificador. La primera función descarga al Presidente de detalles y actividades secundarias; la segunda, le prepara y ordena las actividades fundamentales a realizar (19).

(19) A este respecto, es conveniente conocer las «Reglas de Oro del Secretario», según A. Brauman, que sintetizan el contenido del cargo:

- Misión del Secretario: secundar al jefe.

- Conocer su cargo para ejercerlo y mantenerse en él. varla.

- No es suficiente ganarse la confianza del jefe; es necesario conser-

- Antes de empezar un trabajo, comprenderlo.

- Para ganar tiempo, reflexionar antes de empezar el trabajo.

- Emprender un trabajo por encima de las posibilidades, es exponerse a un fracaso.

- Un trabajo útil no es nunca humillante.

- Anotarlo tode, fecharlo todo, clasificarlo todo, no dejar nada al azar.

- No creerse insustituíble; hacerse indispensable. (Guia de la Secretaria, págs. 84 y 85. Barcelona, 1958.) 


\section{CONCLUSIONES}

Podemos concretar el examen realizado en los siguientes puntos:

1. El Secretario particular de los Presidentes de las Corporaciones locales, es su primer auxiliar en las tareas no directivas. En tanto que en las directivas, el primer colaborador es el Secretario general de la respectiva Corporación.

2. Las funciones principales del Secretario particular son: recepción, preparación, despacho y envío de la correspondencia no oficial; recepción, atención, introducción y despedida de las visitas; atención al teléfono; dirección de la Secretaría particular como unidad administrativa y programación del trabajo del respectivo Presidente.

3. La correspondencia, como vehículo principal de comunicación, debe ser atendida con especial cuidado por el Secretario, con el fin de que resulte un instrumento útil y eficaz de relación exterior de su respectivo Presidente.

4. Las visitas y audiencias demandan una atención primordial del Secretario, con objeto de que no dificulten la actuación del Presidente, sino que la faciliten y la permitan desarrollarse sin obstáculos ni retrasos.

5. El teléfono, como medio de relación personal a distancia, ha de ser objeto de una constante preocupación del Secretario, para conseguir en todo momento que sea el instrumento rápido y eficaz de comunicación.

6. La organización y funcionamiento de la Secretaria particular, como unidad administrativa, requiere de su titular saber estructurarla y dirigirla en la forma más adecuada para alcanzar los cometidos que le corresponden.

7. La programación del trabajo del Presidente demanda del Secretario compenetración con éste, y conocer las actividades que el mismo tiene que realizar y recordarlas en el momento oportuno. 